\title{
APLICACIÓN DE LAS NIIF PARA PYMES EN PERÚ: DIFICULTADES Y DESAFÍOS
}

\author{
APPLICATION OF IFRS FOR SMES IN PERU: \\ DIFFICULTIES AND CHALLENGES
}

Luis Fernando Castro S.

\section{Resumen:}

En el año 2011, según el Consejo Normativo de Contabilidad, entró en vigencia la aplicación obligatoria de las NIIF para PYMES, encargando que se efectúe la difusión y capacitación, entre otros, a las Facultades de Ciencias Contables y Financieras de las universidades del país.

Sin embargo han pasado casi tres años y dentro de los currículos de las universidades no se observa que se hayan incluido estas normas, y esto se refleja en las encuestas realizadas a los contadores de la provincia de Arequipa, que en la mayoría de casos desconocen los alcances de estas NIIF para PYMES.

Es necesario tomar conciencia de la importancia de la aplicación de las NIIF para PYMES, a fin de presentar información financiera relevante y fehaciente.

Palabras Clave: NIIF, PYMES, aplicación, dificultades

\section{Summary:}

In 2011, the Accounting Standards Board put into effect the mandatory application of IFRS for SMEs, ordering its dissemination and training to take place within all the universities in Peru which contain a Faculty of Accounting and Financial Sciences.

However, almost three years after the mandate, there is still no evidence of any Peruvian university incorporating these standards into their curricula. These findings are reflected in 
surveys that given to accountants in the province of Arequipa, which in the majority of the cases are unaware of the full scope of the IFRS as they apply to SMEs.

In order to be able to present relevant and reliable financial information, it is imperative that we are completely aware of how important the implementation of the IFRS for SMEs actually is.

Keywords: IFRS, SMEs, application, difficulties

En las últimas décadas, la contabilidad en Perú ha tomado el rumbo señalado por la globalización y la armonización contable, por ello en nuestro país la profesión contable tiene un marcado protagonismo dentro del quehacer empresarial. Los estados financieros inicialmente fueron desarrollados sobre un contexto de Principios de Contabilidad Generalmente Aceptados, luego se observa un cambio al ser exigible el uso de las Normas Internacionales de Contabilidad para la elaboración de estos informes contables, sobre todo cuando entra en vigencia la Ley General de Sociedades, la misma que establece que los estados financieros se preparan y presentan de conformidad con los Principios de Contabilidad Generalmente Aceptados, esto es, sobre el entorno de Normas Internacionales de Contabilidad oficializadas por el Consejo Normativo de Contabilidad.

Tema aparte son las Micro y Pequeñas Empresas (MYPE), las cuales, según algunas estadísticas, son 3.2 millones de empresas que representan aproximadamente el $97 \%$ del total de empresas peruanas. En muchos de estos negocios se hace imperativo el uso de normatividad contable resumida o sim- plificada, en nuestro caso corresponde aplicar las Normas Internacionales de Información Financiera para Pequeñas y Medianas Empresas.

Habiendo el Consejo Normativo de Contabilidad oficializado en el año 2009 las NIIF para PYMES y siendo su uso obligatorio a partir del año 2011, es necesario realizar un balance de la situación actual de la aplicación de estas normas en las MYPES de nuestro país, y para ello se plantean las siguientes interrogantes:

¿Cuáles son las bases normativas que obligan a las empresas a presentar su información financiera en base a las NIIF para PYMES?

¿Cuál es el grado de conocimiento y aplicación de las NIIF para PYMES?

¿Cuáles son las principales dificultades al aplicar o tratar de aplicar las NIIF para PYMES?

¿Qué desafíos tienen las Facultades de Ciencias Contables y Financieras de las universidades peruanas?

Realizando una verificación rápida de material bibliográfico vinculado a las NIIF 
para PYMES, de las universidades de esta localidad (provincia de Arequipa - Perú), se observa que se cuenta con escasos libros sobre el tema planteado; sin embargo, existe regular cantidad de libros relacionados a la aplicación de las NIIF. Este primer acercamiento motiva la realización de esta sucinta investigación denominada "Aplicación de las NIIF para PYMES en el Perú: Dificultades y Desafíos", la misma que está circunscrita a la región Arequipa y, específicamente, a la provincia de Arequipa.

A fin de responder a las interrogantes, se han planteado los siguientes objetivos:

- Analizar las bases normativas que obligan que los estados financieros sean presentados y elaborados en base a las Normas Internacionales de Información Financiera para Pequeñas y Medianas Empresas.

- Determinar el grado de conocimiento de las NIIF para PYMES y la aplicación de éstas en la elaboración de estados financieros por parte de los Contadores Públicos de la región Arequipa.

- Conocer cuáles son las principales dificultades que tiene el Contador Público al aplicar o tratar de aplicar las NIIF para PYMES en las empresas de la región Arequipa.

- Determinar los desafíos de las Facultades de Contabilidad de las universidades peruanas.

\section{Normatividad relacionada con las NIIF para PYMES}

En primer lugar, se debe indicar que el Sistema Nacional de Contabilidad de Perú cuen- ta con un organismo denominado Consejo Normativo de Contabilidad, que tiene a su cargo el estudio, análisis y emisión de normas en asuntos para los cuales son convocados por el Contador General de la Nación. Este Consejo se reúne dos veces al año y está integrado por representantes de diversas entidades como el Banco Central de Reserva, la Superintendencia de Mercado de Valores, la Superintendencia de Banca y Seguros, la Superintendencia Nacional de Aduanas y de Administración Tributaria, el Instituto Nacional de Estadística, entre otros, así como un representante de la Junta de Decanos de los Colegios de Contadores Públicos y de la Facultad de Ciencias de la Contabilidad de las universidades del país. El Consejo Normativo de Contabilidad tiene su inicio y atribuciones en la Ley $N^{\circ}$ 2468o (Ley del Sistema Nacional de Contabilidad), publicada en junio de $1987 \mathrm{y}$ actualmente se encuentra bajo el marco de la Ley $N^{\circ} 28708$ (Ley General del Sistema Nacional de Contabilidad), publicada en marzo de 2006.

Las principales normas relacionadas con la obligación del contador a observar las Normas Internacionales de Contabilidad y NIIF para PYMES son las siguientes:

\section{Resolución del Consejo Normativo de} Contabilidad $\mathrm{N}^{\circ}$ oo8-97-EF/93.o1: En esta norma se precisa que "es obligación del Contador Público en el ejercicio profesional en función dependiente, observar bajo estricta responsabilidad y criterio profesional la correcta aplicación de los Principios de Contabilidad Generalmente Aceptados, las Normas Internacionales de Contabilidad, reconocidas por la profesión, en los Congresos Nacio- 
nales de Contadores Públicos y oficializadas por el Consejo Normativo de Contabilidad...", asimismo se precisa "como conclusión, que en caso que el Contador Público Colegiado, no cumpla lo prescrito en los artículos anteriores, incurre en el comportamiento de culpa inexcusable a que se refiere el Código Civil".

Resolución del Consejo Normativo de Contabilidad $\mathrm{N}^{\circ}$ o13-98-EF/93.01: Al ser Perú integrante del Comité de Normas Internacionales de Contabilidad (IASC) a través de la Federación Internacional de Contadores (IFAC), y que la Nueva Ley General de Sociedades, en su artículo $223^{\circ}$ señala que los estados financieros se preparan de conformidad con los Principios de Contabilidad Generalmente Aceptados, la resolución precisa "que los Principios de Contabilidad Generalmente Aceptados a que se refiere el Artículo $223^{\circ}$ de la Nueva Ley General de Sociedades comprende, substancialmente, a las Normas Internacionales de Contabilidad (NICs), oficializadas mediante Resoluciones del CNC...."

Resolución del Consejo Normativo de Contabilidad $\mathrm{N}^{\circ}$ o41-98-EF/93.01: $\mathrm{Me}-$ diante esta resolución se aprueba "el uso obligatorio del Plan Contable General Empresarial... que entrará en vigencia a partir del o1 de enero de 2010...", recomendando la difusión entre otros "a las Facultades de Ciencias Contables y Financieras de las Universidades del País".

Resolución del Consejo Normativo de Contabilidad $\mathrm{N}^{\circ}$ o45-98-EF/93.01: Oficializa "la aplicación de la Norma Internacional de Información Financiera para Pequeñas y Medianas Entidades (NIIF para las PYMES)... la misma que es de aplicación obligatoria para las entidades que no tienen obligación pública de rendir cuentas...", señala además que "se encuentran excluidas del ámbito de aplicación de la presente norma las siguientes entidades: a). Las empresas bajo la supervisión y control de la Comisión Nacional Supervisora de Empresas y Valores -CONASEV (hoy Superintendencia de Mercado de Valores) y b). Las empresas que obtengan ingresos anuales por ventas de bienes y/o servicios o tengan activos totales, iguales o mayores, en ambos casos a tres mil (3 ooo) Unidades Impositivas Tributarias, al cierre del ejercicio anterior, a las cuales les son aplicables las NIIF completas, oficializadas por el Consejo Normativo de Contabilidad - CNC...." Esta norma establece que las NIIF para PYMES “entrará en vigencia a partir del 1 de enero de 2011”. Y por último encarga que se efectúe la difusión y capacitación, entre otra, a las "Facultades de Ciencias Contables y Financieras de las Universidades del país,...”.

En segundo lugar, tenemos la Ley $\mathrm{N}^{\circ} 26887$ (Ley General de Sociedades), la misma que en el artículo $223^{\circ}$ - Preparación y presentación de Estados Financieros de la Sección Sexta - Estados Financieros y Aplicación de Utilidades, señala que: "Los estados financieros se preparan y presentan de conformidad con las disposiciones legales sobre la materia y con principios de contabilidad generalmente aceptados en el país."

Por último, tenemos el Decreto Supremo $\mathrm{N}^{\circ}$ 122-94-EF, Reglamento de Ley del Impuesto a la Renta, el mismo que en el literal g) Normas Supletorias, del artículo $11^{\circ}-$ Costo Computable inserto en el Capítulo V - De 
la Renta Bruta, establece que "Para la determinación del costo computable de los bienes o servicios, se tendrán en cuenta supletoriamente las normas que regulan el ajuste por inflación con incidencia tributaria, las Normas Internacionales de Contabilidad y los principios de contabilidad generalmente aceptados, en tanto no se opongan a lo dispuesto en la Ley y en este Reglamento". Asimismo en el artículo $33^{\circ}$ - Diferencias en la determinación de la Renta Neta por la aplicación de Principios de Contabilidad Generalmente Aceptados del Capítulo IX - Del Régimen para Determinar la Renta, se establece que "La contabilización de operaciones bajo principios de contabilidad generalmente aceptados, puede determinar, por la aplicación de las normas contenidas en la Ley, diferencias temporales y permanentes en la determinación de la renta neta. En consecuencia, salvo que la Ley o el Reglamento condicionen la deducción al registro contable, la forma de contabilización de las operaciones no originará la pérdida de una deducción. Las diferencias temporales y permanentes obligarán al ajuste del resultado según los registros contables, en la declaración jurada".

\section{Aplicación de las NIC - NIIF en Perú}

Como se puede apreciar del punto anterior, el Consejo Normativo de Contabilidad ha oficializado en su momento las NIC y posteriormente las NIIF, y al mismo tiempo ha modificado el Plan Contable concordándolo con las NIIF, era necesario este cambio. Al mismo tiempo, otras normas como la Ley
General de Sociedades y la Ley del Impuesto a la Renta también han considerado algunos puntos sobre la aplicación de las normas internacionales, este itinerario seguido por las NIIF, básicamente por las Resoluciones del $\mathrm{CNC}$, obligan a los contadores a estar, en primer lugar, actualizados en temas de normatividad contable, y en segundo lugar, aplicarlo en las empresas dependiendo del volumen de ingresos o monto de activos fijos.

Actualmente la aplicación de las NIC - NIIF tiene tres escenarios, tal como se muestra en el siguiente Cuadro $\mathrm{N}^{\circ}$ :

\section{Cuadro $\mathrm{N}^{\circ} 1$}

Aplicación de las NIC - NIIF en Perú

\begin{tabular}{l|c|l}
\hline \multicolumn{1}{c|}{ NIC - NIIF } & $\begin{array}{c}\text { AÑO DE } \\
\text { APLICACIÓN } \\
\text { PLENA }\end{array}$ & VERSIÓN A APLICAR \\
\hline $\begin{array}{l}\text { NIC - NIIF FULL para } \\
\text { empresas que cotizan en } \\
\text { Bolsa y empresas con } \\
\text { Responsabilidad Pública. }\end{array}$ & 2011 & $\begin{array}{l}\text { Versión actualizada por } \\
\text { Fundación IFRS (IASB) con } \\
\text { valor normativo. }\end{array}$ \\
\hline $\begin{array}{l}\text { NIC - NIIF para empresas } \\
\text { que superen 3,ooo UIT en } \\
\text { ventas o activos totales el } \\
\text { 2010. }\end{array}$ & 2011 & $\begin{array}{l}\text { Versión 2012 de NIC - NIIF, } \\
\text { oficializada con Resolución } \\
\mathrm{N}^{\circ} \text { 051-2012-EF/30. }\end{array}$ \\
\hline $\begin{array}{l}\text { NIIF para PYMES, para } \\
\text { empresas que no superen } \\
\text { 3,ooo UIT en ventas o } \\
\text { activos totales el 2o10. }\end{array}$ & 2011 & $\begin{array}{l}\text { Versión 2009, no modificada } \\
\text { a la fecha, estable hasta 2014. }\end{array}$ \\
\hline
\end{tabular}

FUENTE: Consejo Normativo de Contabilidad.

Elaboración Propia

Si analizamos cuántas empresas tendrían que adecuar su contabilidad a NIC - NIIF, y teniendo como referencia los regímenes tributarios establecidos por la normatividad tributaria en Perú, tenemos que aproximadamente 7000 empresas deberían usar NIC - NIIF completas (aprobadas por el CNC) y alrededor de 500,000 empresas las NIIF para PYMES. Veamos el Cuadro $\mathrm{N}^{\circ}{ }_{2}$ : 


\section{Cuadro $\mathrm{N}^{\circ} 2$}

Regímenes tributarios y aplicación

de NIC - NIIF

\begin{tabular}{|l|c|l|}
\hline RÉGIMEN TRIBUTARIO & $\begin{array}{l}\text { CANTIDAD DE } \\
\text { EMPRESAS } \\
\text { (Aproximado) }\end{array}$ & \multicolumn{1}{|c|}{ CONTABILIDAD } \\
\hline $\begin{array}{l}\text { Régimen General: } \\
\text { Ingresos mayores a 3,00o } \\
\text { UIT. }\end{array}$ & 7,000 & $\begin{array}{l}\text { NORMAS NIIF COMPLETAS } \\
- \text { NIIF FULL } \\
(2 \%)\end{array}$ \\
\hline $\begin{array}{l}\text { Régimen General: } \\
\text { Ingresos menores a 3,00o } \\
\text { UIT. }\end{array}$ & 500,000 & $\begin{array}{l}\text { NORMAS NIIF PARA PYMES } \\
- \text { NIIF PYMES } \\
(98 \%)\end{array}$ \\
\hline $\begin{array}{l}\text { Régimen Especial de } \\
\text { Renta - RER }\end{array}$ & RER & $\begin{array}{l}\text { SÓLO LLEVAN REGISTROS } \\
\text { DE VENTAS Y COMPRAS }\end{array}$ \\
\hline $\begin{array}{l}\text { Nuevo Régimen Único } \\
\text { Simplificado }\end{array}$ & NRUS & $\begin{array}{l}\text { NO OBLIGADOS A LLEVAR } \\
\text { CONTABILIDAD }\end{array}$ \\
\hline
\end{tabular}

Fuente: Declaraciones Juradas Anuales 2011 - SUNAT.

Elaboración Propia

Por otro lado, considerando los cambios en las normas internacionales y el Plan Contable General Empresarial, la Administración Tributaria dictó diversas normas a fin de ir adecuando sobre todo los Libros Contables vinculados a asuntos tributarios con las obligaciones contables, emitiéndose la Resolución de Superintendencia $\mathrm{N}^{\circ}$ 234-2006/ SUNAT (Formato de los Libros Físicos) y posteriormente la Resolución de Superintendencia $\mathrm{N}^{\circ}$ 286-2009/SUNAT (Libros Electrónicos) y otras normas complementarias a estas, que buscan adecuar los formatos al Plan Contable General Empresarial y a las Normas Internacionales de Información Financiera.

Si continuamos con el análisis de los libros y registros contables y sabiendo que SUNAT reguló el uso y los formatos de estos libros, veamos la incidencia en las Normas Internacionales de Contabilidad en el Cuadro $\mathrm{N}^{\circ} 3$.

\author{
Cuadro $\mathrm{N}^{\circ} 3$ \\ NIC - NIIF y libros y registros \\ contables tributarios
}

Fuente: SUNAT.

Elaboración propia

Respecto al cuadro $\mathrm{N}^{\circ}$, se debe tener en cuenta que en el tramo de más de 3,00o UIT, a la contabilidad se le agrega los Registros de Costos y el Registro de Inventario Permanente Valorizado. En el tramo de 150 a 3,00o UIT, existen tres tramos: el primer tramo hasta 500 UIT, los cuales elaboran un inventario anual; el segundo tramo hasta 1,50o UIT, los que están obligados a llevar un Registro de Inventario Permanente en Unidades Físicas; y el tercer tramo más de 1,500 UIT, que deben llevar Registros de Costos y el Registro de Inventario Permanente Valorizado.

Asimismo se debe tener presente que la Administración Tributaria, dentro del Código Tributario, ha establecido Infracciones y Sanciones vinculadas con la obligación de llevar libros y registros contables, en el Artículo $175^{\circ}$ del referido cuerpo normativo, y en específico el numeral 2 del referido artículo, señala que constituye una infracción tributaria: "Llevar los libros de contabilidad y otros libros y registros exigidos por la leyes, reglamentos o por Resolución de Superintendencia de la SUNAT; sin observar las formas y condiciones establecidas en las normas correspondientes". La sanción establecida en 
las tablas es de $0.3 \%$ de los Ingresos Netos, la misma que no podrá ser menor al $10 \%$ de la UIT (S/. 370.00), ni mayor de 12 UIT (S/. 44,400.00).

Según lo establecido en el párrafo anterior, podríamos inferir que la empresa que no cumple con llevar la contabilidad simplificada o completa, o no usa el Plan Contable General Empresarial o no aplica las NIIF para PYMES o NIIF completas, podrían ser pasibles de ser sancionados por este hecho desde un punto de vista tributario.

Haciendo un recuento de las disposiciones contables, realmente no encontramos un elemento coercitivo para la aplicación de las NIC - NIIF o las NIIF para PYMES, pese a las disposiciones emanadas de la Superintendencia de Mercado de Valores, éstas van direccionadas básicamente a las empresas que cotizan en la Bolsa de Valores, cuyos estados financieros deben ser presentados auditados, al igual que aquellas empresas que superan las 3,00o UIT pero no cotizan en bolsa, tienen la necesidad de presentar estados financieros auditados, según la Resolución SMV Nº11-2012-SMV/o1. Se debe tener presente que la Administración Tributaria ha establecido infracciones y sanciones en caso la empresa omita llevar la contabilidad en consideración con las normas y leyes respectivas.

\section{Resultados}

Saber qué porcentaje de contadores públicos conocen y aplican las NIIF para PYMES es un tema relevante en la presente investigación, debido a que no solo nos centrare- mos en la parte normativa sino que, parte de los objetivos de este trabajo, es determinar el grado de conocimiento de las NIIF para PYMES, y para ello se ha preparado una encuesta.

A fin de responder a las interrogantes planteadas, se realizó una encuesta a un total de 120 contadores escogidos al azar de un total de 5118 colegiados, a los cuales se les aplicó una batería de 9 preguntas, de las cuales se obtuvo el siguiente resultado:

Pregunta $\mathrm{N}^{\circ} 1$

$¿$ Es usted contador Dependiente o Independiente?

Esta pregunta se formula a fin de relacionar el conocimiento de la Norma Internacional de Información Financiera para PYMES respecto al vínculo laboral con las empresas, y ver si existe alguna variación sustancial respecto a la aplicación de esta NIIF.

\section{Cuadro $\mathrm{N}^{\circ} 4$}

Régimen Laboral del Contador

\begin{tabular}{|c|c|c|}
\hline & Cantidad & $\%$ \\
\hline Dependiente & 73 & $60.83 \%$ \\
\hline Independiente & 47 & $39.17 \%$ \\
\hline TOTAL & 120 & $100.00 \%$ \\
\hline
\end{tabular}

Se observa que el 61\% de los contadores encuestados laboran de manera dependiente, lo que podría evidenciar que nos encontramos frente a profesionales de empresas cuyos ingresos son iguales o mayores a las 3000 
UIT y cuyo conocimiento de las NIIF debería ser más sólido, por tanto, es probable que este grupo de contadores tenga poco interés en las NIIF para PYMES y esté más abocado a aplicar las NIIF completas. El 39\% de encuestados refiere laborar de manera independiente y la probabilidad de que las empresas a las cuales asesoren tengan ingresos menores a 3000 UIT es muy alta.

\section{Pregunta $\mathrm{N}^{\circ} 2$}

¿Cuál es el volumen de ingresos y activos de la empresa en la cual labora como contador? Esta pregunta permitirá sesgar el número de entrevistados, dedicándonos exclusivamente a aquellos contadores cuyas empresas tengan ingresos y activos menores a 3000 UIT.

\section{Cuadro $\mathrm{N}^{\circ} 5$}

Dependientes: Ingresos de Empresa

\begin{tabular}{|c|c|c|}
\hline & Cantidad & $\%$ \\
\hline Más de 300o UIT & 28 & $38.36 \%$ \\
\hline Menos de 300o UIT & 45 & $61.64 \%$ \\
\hline TOTAL & 73 & $100.00 \%$ \\
\hline
\end{tabular}

Del total de 73 contadores que laboran en diversas empresas de manera dependiente, 45 contadores trabajan en empresas cuyos ingresos o activos anuales son menores a 3000 UIT, este grupo de contadores debería usar las NIIF para PYMES; al restante $38 \%$ de contadores que laboran en empresas cuyos ingresos o activos anuales son iguales o mayores a 3000 UIT, les corresponde usar las NIC y NIIF completas publicadas por el Consejo Normativo de Contabilidad.
Pregunta $\mathrm{N}^{\circ} 3$

¿Cuál es el volumen de ingresos y activos de las empresas a las cuales asesora usted como contador independiente?

Esta pregunta permitirá sesgar el número de entrevistados, dedicándonos exclusivamente a aquellos contadores cuyas empresas tengan ingresos y activos menores a 3000 UIT.

\section{Cuadro $\mathrm{N}^{\circ} 6$}

Independientes: Ingreso de Empresa

\begin{tabular}{|c|c|c|}
\hline & Cantidad & $\%$ \\
\hline Más de 30oo UIT & 2 & $4.26 \%$ \\
\hline Menos de 30oo UIT & 45 & $95.74 \%$ \\
\hline TOTAL & 47 & $100.00 \%$ \\
\hline
\end{tabular}

Del total de 47 contadores que laboran de manera independiente, generalmente en estudios contables, 45 contadores asesoran a empresas cuyos ingresos anuales son menores a 3000 UIT, este grupo de contadores debe usar las NIIF para PYMES; el restante 4\% (2 contadores) llevan contabilidades a empresas cuyos ingresos y activos anuales son iguales o mayores a 3000 UIT, y les corresponde usar las NIC y NIIF completas publicadas por el Consejo Normativo de Contabilidad, este grupo de contadores son retirados de la muestra.

\section{Pregunta $\mathrm{N}^{\circ} 4$}

¿Conoce usted las NIIF para PYMES?

Cuestionamiento directo a fin de conocer el número de entrevistados que tienen conocimientos de las NIIF para PYMES y señalar cuántos son contadores dependientes e independientes. 
Cuadro $\mathrm{N}^{\circ} 7$

Conoce las NIIF para PYMES

\begin{tabular}{|c|c|c|}
\hline & Cantidad & $\%$ \\
\hline Sí & 30 & $33.33 \%$ \\
\hline NO & 60 & $66.67 \%$ \\
\hline TOTAL & 90 & $100.00 \%$ \\
\hline
\end{tabular}

Del total de contadores cuyos ingresos son menores a 3000 UIT (9o profesionales), el $33 \%$ conoce las NIIF para PYMES (esto representa aproximadamente 30 contadores); sin embargo, el $67 \%$ restante manifiesta no conocer las NIIF para PYMES, por lo tanto la probabilidad de aplicación de esta norma es remota. Es necesario indicar que de 30 contadores que señalaron conocer las NIIF para PYMES, 13 son dependientes y 17 independientes, responde casi a la misma proporción de contadores dependientes y contadores independientes, por lo que resulta irrelevante la condición laboral del contador para la presente investigación, quedándonos sólo con la cantidad de contadores que conoce las NIIF para PYMES.

\section{Pregunta $\mathrm{N}^{\circ} 5$}

¿Sabe desde cuándo están vigentes las NIIF para PYMES?

Si bien el entrevistado dice conocer las NIIF para PYMES, es necesario hacer algunas preguntas para determinar la realidad de las respuestas, como en este caso.

\section{Cuadro $\mathrm{N}^{\circ} 8$}

¿Sabe desde cuándo está vigente?

\begin{tabular}{|c|c|c|}
\hline & Cantidad & $\%$ \\
\hline SÍ & 26 & $86.67 \%$ \\
\hline NO & 4 & $13.33 \%$ \\
\hline TOTAL & 30 & $100.00 \%$ \\
\hline
\end{tabular}

Ante la consulta, 26 de los encuestados manifestaron saber desde cuándo se encuentran vigentes las NIIF para PYMES, esto representa el $87 \%$ del total de profesionales que dijeron conocer las NIIF para PYMES; el restante $13 \%$, pese a conocer las NIIF para PYMES, refieren no saber desde cuándo está vigente la norma. Si bien la respuesta es directa es necesario confirmar este dato, a fin de no distorsionar los resultados; por tal motivo, se ha visto por conveniente formular la siguiente interrogante, que nos permitirá saber con mayor precisión si conocen las NIIF para PYMES.

\section{Pregunta 6}

Indique usted ¿desde qué año están vigentes las NIIF para MYPES?

Esta pregunta nos permitirá conocer objetivamente cuántos entrevistados conocen las NIIF.

\section{Cuadro $\mathrm{N}^{\circ} 9$}

¿Desde qué año?

\begin{tabular}{|c|c|c|}
\hline & Cantidad & $\%$ \\
\hline OTRO AÑO & 16 & $53.33 \%$ \\
\hline TOTAL & 30 & $46.67 \%$ \\
\hline
\end{tabular}


La respuesta correcta es que la vigencia de las NIIF para PYMES es desde el año 2011, sin embargo, sólo 16 entrevistados contestaron afirmativamente, representando el $53 \%$ del total de contadores que indicaron conocerlas, asimismo señalaron saber desde cuándo estaban vigentes en Perú; el restante $47 \%$ señaló otras fechas de entrada en vigencia, lo que evidencia que si bien pueden conocer las NIIF para PYMES desconocen la entrada en vigencia de las normas en nuestro país. Esta pregunta nos permite determinar y separar de la muestra a aquellos contadores que no conocen esta norma.

\section{Pregunta $\mathrm{N}^{\circ} 7$}

¿Aplica usted las NIIF para PYMES en su empresa?

Después de haber determinado la cantidad de entrevistados que conocen las NIIF y saben desde cuándo es su vigencia, observaremos el número de contadores que aplican estas normas.

\section{Cuadro $\mathrm{N}^{\circ} 10$}

¿Aplica las NIIF para PYMES

en su empresa?

\begin{tabular}{|c|c|c|}
\hline & Cantidad & $\%$ \\
\hline SÍ & 11 & $36.67 \%$ \\
\hline NO & 19 & $63.33 \%$ \\
\hline TOTAL & 30 & $100.00 \%$ \\
\hline
\end{tabular}

Del total de entrevistados que indicaron saber desde cuándo están vigentes las NIIF para PYMES en Perú, aproximadamente el $37 \%$ señaló que vienen aplicando estas normas en sus respectivas empresas, lo que re- presenta 11 profesionales; el restante $63 \%$ refiere conocer las normas pero no aplicarlas, evidencia un claro problema al momento de elaborar los estados financieros de las empresas, los mismos que no se encontrarían armonizados con las NIIF para PYMES.

\section{Pregunta $\mathrm{N}^{\circ} 8$}

En caso de que la empresa en la cual usted labora o asesora tenga ingresos o activos iguales o mayores a 3000 UIT ¿aplica la NIIF oficializada por el Consejo Normativo de Contabilidad?

Si bien no es parte de la investigación, esta pregunta nos permitirá determinar el porcentaje de aplicación de la NIIF completa.

\section{Cuadro $\mathrm{N}^{\circ} 11$}

Aplicación NIC-NIIF COMPLETA

\begin{tabular}{|c|c|c|}
\hline & Cantidad & $\%$ \\
\hline SÍ & 11 & $36.67 \%$ \\
\hline NO & 19 & $63.33 \%$ \\
\hline TOTAL & 30 & $100.00 \%$ \\
\hline
\end{tabular}

Del total de profesionales entrevistados, 30 de ellos manifestaron laborar en empresas cuyos ingresos anuales son iguales o superiores a 3000 UIT; sin embargo, sólo el 37\% (11 contadores) indicó que en sus empresas se están aplicando las NIC - NIIF de manera integral; y el restante $63 \%$ (19 profesionales) refiere que no están aplicando estas normas, tal como lo establece el Consejo Normativo de Contabilidad. Como se puede apreciar, el porcentaje es el mismo para aquellos entrevistados que tienen la obligación de elaborar estados financieros en base a las NIIF para MYPES. 
Pregunta $\mathrm{N}^{\circ} 9$

¿Qué dificultades considera que existen para poder aplicar las NIIF para PYMES en la región?

\section{Cuadro $\mathrm{N}^{\circ} 12$}

\begin{tabular}{|l|c|c|}
\hline & Cantidad & $\%$ \\
\hline No hay Cursos, Seminarios, Talleres, etc. & 36 & $40.00 \%$ \\
\hline La norma no es de fácil comprensión & 24 & $26.67 \%$ \\
\hline En la Universidad nunca enseñaron su aplicación & 18 & $20.00 \%$ \\
\hline No hay información contable fiable & 6 & $6.67 \%$ \\
\hline No se cuentan con sistemas adecuados & 3 & $3.33 \%$ \\
\hline Es muy costosa su aplicación & 3 & $3.33 \%$ \\
\hline TOTAL & 90 & $100 \%$ \\
\hline
\end{tabular}

Los entrevistados refieren que las dificultades que encontraron al momento de aplicar las NIIF para PYMES en las empresas, responden básicamente a un problema de orden académico, falta de capacitación o entrenamiento formal (Diplomados, Especialización, Cursos, Seminarios, Talleres, etc.); asimismo, de alguna manera involucran, en parte, el trabajo de la Universidad respecto al conocimiento de las NIIF para PYMES, aunque otro grupo refiere que la referida NIIF no es de fácil comprensión.

Al observar de manera integral los resultados, podemos determinar que de un total de 90 profesionales entrevistados, cuyas empresas tienen ingresos anuales menores a 3,0oo UIT, aproximadamente sólo el 18\% (16 contadores) conoce las NIIF para PYMES; y sin embargo, sólo 11 contadores indicaron que aplican las NIIF para PYMES en sus respectivas empresas, lo que representa un $12 \%$ del total de profesionales que indicaron laborar o asesorar a empresas del nivel de ingresos o activos menores a 3000 UIT. Este hecho se replica en aquellos entrevis- tados que indicaron que las empresas en las cuales laboran o asesoran tienen ingresos o activos iguales o mayores a 3000 UIT, comparado con el porcentaje de contadores que conocen y aplican las NIIF para PYMES.

Analizando los resultados de las encuestas podemos determinar que el grado de conocimiento y aplicación de las NIIF para PYMES y de la NIIF oficializada por el CNC es el mismo; en ambos casos el porcentaje de entrevistados es coincidente, 37\% del total de contadores de empresas cuyos ingresos o activos son superiores a 3000 UIT aplican la NIIF completa y el mismo porcentaje de los contadores que conocen las NIIF para PYMES la aplican en sus respectivas empresas.

Es evidente que las posibles dificultades estén relacionadas con el desconocimiento de las NIIF para PYMES, al señalar como una causa principal la falta de capacitación en estas normas, lo que puede ser consecuente con el segundo motivo de dificultad señalado por los entrevistados, esto es, el problema de comprensión de las NIIF para PYMES.

\section{Discusión}

Al analizar las razones por las cuales los contadores desconocen la forma de aplicación de las NIIF para PYMES, encontramos la poca difusión de la norma y la escasa o nula implementación de Cursos o Talleres de los colegios profesionales de contadores así como de las universidades de la región, y esto básicamente debido a que los contadores aún están basando la contabilidad en 
conceptos tributarios, es decir, desarrollando la contabilidad desde un punto de vista íntegramente tributario, teniendo en cuenta que el incumplimiento de disposiciones tributarias trae como consecuencia sanciones de carácter administrativo, que pueden lacerar la economía de una empresa.

Por otro lado, a las empresas les dificulta implementar sistemas informáticos que permitan un rápido y ágil procesamiento de la información contable; quizás con la última implementación de Libros y Registros Electrónicos por parte de la Administración Tributaria, los empresarios cambien la forma de enfocar la contabilidad y la hagan considerando los avances tecnológicos; el tener la contabilidad manual (en libros preimpresos), dificulta el trabajo del contador al momento de querer elaborar los estados financieros de la empresa.

El entrenamiento de los contadores requiere una inversión muy elevada en promedio. Un Programa de Alta Especialización en las NIIF tiene un costo aproximado de 3,200 dólares americanos y una duración aproximada de siete a ocho meses, resultando por tanto una fuerte inversión en capacitación para poder contar con profesionales expertos en temas de NIIF.

Existe un desconocimiento por parte de los contadores y empresarios sobre las disposiciones del Consejo Normativo de Contabilidad y resoluciones de la Superintendencia de Mercado de Valores, a tal punto de pensar que una pequeña empresa es aquella cuyos ingresos anuales no superan las 150 UIT (S/. 555,00o), por tanto, desconocen la Resolu- ción del CNC, el mismo que establece que las NIIF para PYMES se usa en empresas cuyos ingresos o activos anuales no superan las 3,00o UIT (S/. 11'100,00o); es más, la contabilidad es procesada en libros y registros contables vinculados a asuntos tributarios, en algunos casos exclusivamente tributarios, descuidando la información contable necesaria para la toma de decisiones.

En la región Arequipa se cuenta con tres universidades, y según la verificación a los sílabos, sólo en una de ellas se ha considerado la incorporación de las NIC - NIIF de manera agresiva durante todo el período de formación del contador, y en las dos universidades restantes se trabajan las NIC - NIIF sólo en algunos cursos de carrera, privilegiando el conocimiento tributario más que el contable. Sin embargo, en todas ellas no se ha establecido un apartado para conocer los alcances de las NIIF para PYMES, por tanto es evidente que las respuestas de los consultados son contundentes, no se conocen los lineamientos de las NIIF para este sector económico.

Durante el año 2010, el Programa Profesional de Contabilidad dio inicio a un proceso de restructuración de la malla curricular, realizando cambios e incorporaciones en los cursos de carrera a fin de hacer más atractiva y acorde a los nuevos cambios en materia contable que se implementaron (oficialización de las NIC, NIIF, SIC y CINIIF, y el uso obligatorio del Plan Contable General Empresarial).

Esta malla curricular entra en vigencia el año 2011, al mismo tiempo que entran en vi- 
gencia el uso obligatorio de las NIC - NIIF y el Plan Contable General Empresarial, por tanto se realizan ciertos ajustes a los cursos de carrera introduciendo las actuales Normas Internacionales de Información Financiera (NIIF) sin esperar que estas sean abordadas y estudiadas "exclusivamente" en un par de cursos. Esto en consideración a que el futuro profesional contable debe familiarizarse y conocer desde su ingreso al Programa de Contabilidad, la existencia y aplicación de las NIIF, por tal motivo se incorporan en los distintos sílabos de los cursos de carrera las NIIF pertinentes estudiándose de manera progresiva, tal como se muestra en el diseño siguiente (Gráfico $\mathrm{N}^{\circ}{ }_{1}$ ). Esta forma de trabajo tiene un solo objetivo, lograr que el egresado (contador público) tenga sólidos conocimientos de las Normas Internacionales de Información Financiera (NIIF) y cuente con las herramientas y conocimientos necesarios para lograr su aplicación en la empresa.

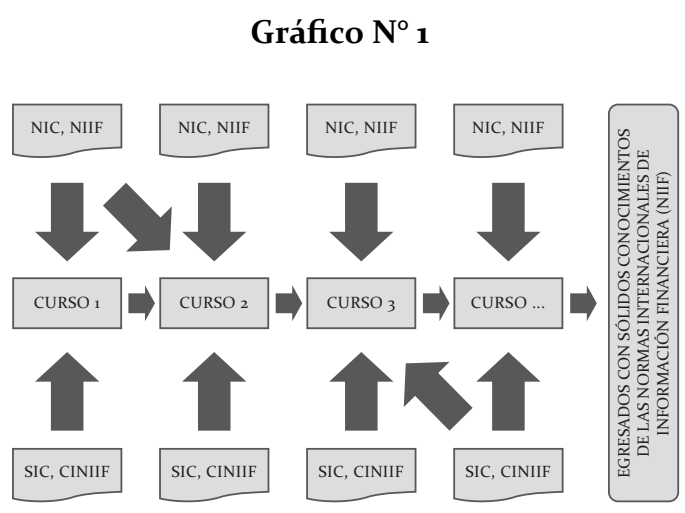

El gráfico anterior muestra cómo se relacionan actualmente los cursos de carrera (Fundamentos de Contabilidad, Contabilidad Intermedia, etc.) con las NIC y NIIF $\mathrm{y}$ sus correspondientes interpretaciones, las SIC y CINIIF, con la intención de dar a los estudiantes conocimientos de la aplicación práctica de las normas contables, más aun sabiendo que el Concejo Normativo de Contabilidad ha declarado a las Normas Internacionales de Información Financiera como los Principios de Contabilidad Generalmente Aceptados.

Por otro lado, no hemos perdido de vista el hecho de que el actual Plan Contable General Empresarial tiene su base en las Normas Internacionales de Información Financiera (NIC, NIIF, SIC y CINIIF), y en la dinámica de cada cuenta se ha incluido las NIIF e interpretaciones relacionadas a las cuentas. Son estos hechos los que nos conducen a incluir en nuestros cursos de carrera cimientos necesarios para el futuro desempeño eficiente del Contador egresado de nuestra casa de estudios.

Es por esto que se han incluido en los sílabos referencias a las Normas Internacionales de Información Financiera vinculadas al curso que desarrolla el docente, y que dentro de la Bibliografía necesariamente se debe incluir el Libro del IFRS, partes A y B. Presento en los siguientes cuadros algunos ejemplos de cursos en los cuales se estudian específicamente algunas NIC - NIIF. 


\section{Cuadro $\mathrm{N}^{\circ} 13$}

Incorporación de NIC - NIIF

en cursos de carrera

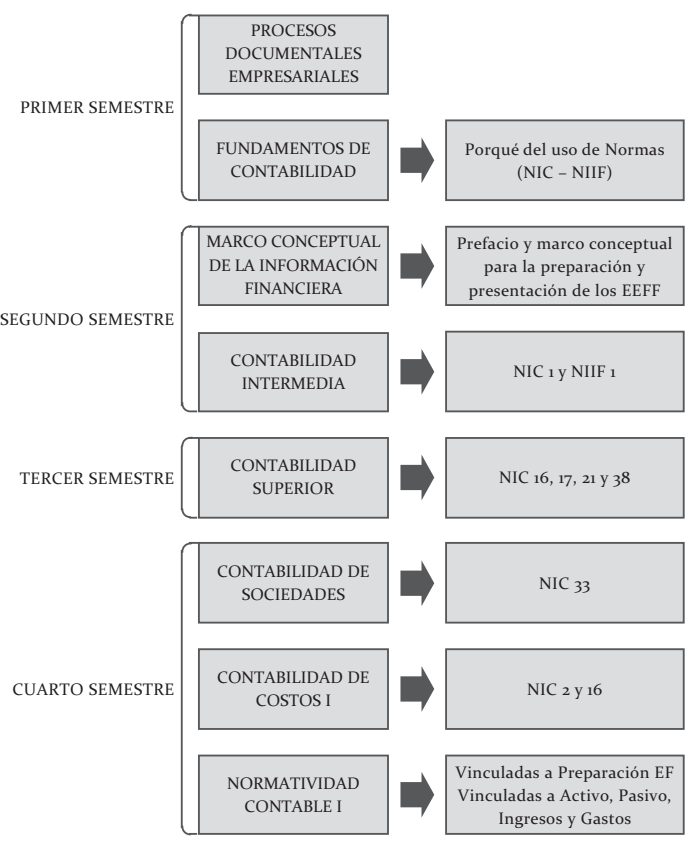

\section{Conclusiones y recomendaciones}

Podemos establecer, a modo de conclusiones:

- Según el Consejo Normativo de Contabilidad, en caso de no aplicar las NIC- NIIF el Contador Público incurre en comportamiento de culpa inexcusable; y la aplicación de las NIC - NIIF y NIIF para PYMES entró en vigencia desde el or de enero de 2011.

- A partir de la encuesta aplicada, se concluye que existe desconocimiento de los alcances de las NIIF para PYMES, por tanto la aplicación de esta norma en las pequeñas y medianas empresas es casi nulo, más aún sabiendo que el contador principalmente privilegia la normatividad tributaria por el grado coercitivo que tiene en las empresas; se puede decir que la contabilidad empresarial está preparada para fines tributarios casi de manera exclusiva, por tanto no aplican la normatividad contable.

- La principal dificultad que tiene el contador para poder aplicar las NIIF para PYMES, es la falta de entrenamiento formal; no se observa que los colegios profesionales ni las universidades hayan realizado cursos, seminarios, talleres, entre otros, para poder dar soporte al trabajo de los contadores públicos.

Asimismo planteo las siguientes recomendaciones:

- Es necesaria la elaboración de un programa de entrenamiento en NIIF para PYMES en los colegios profesiones, los cuales son los llamados a realizar este tipo de eventos, a modo de Congresos, Seminarios Internacionales, etc., a fin de difundir con mayor agresividad los alcances de estas normas.

- Se requiere un trabajo conjunto entre los órganos reguladores (CNC, SMV, Sociedades de Auditoría, etc.) y las universidades a fin de intercambiar experiencias a nivel nacional e internacional que puedan ser replicadas en los salones de estudio y elevar el número de empresas que apliquen las NIIF.

- Se debería estandarizar las mallas curri- 
culares de las Facultades de Contabilidad del país, a fin de elevar el nivel profesional de los egresados de los Programas de Contabilidad; se debe tener siempre presente que en todas las Resoluciones del Consejo Normativo de Contabilidad sobre la oficialización de las Normas Internacionales de Información Financiera, encargan a las Facultades de Contabilidad de las universidades del país la difusión y capacitación de las referidas normas, tanto las NIC - NIIF así como las NIIF para PYMES.

\section{Referencias}

Apaza Meza, Mario (2011). "Análisis de la NIIF para PYMES y casos prácticos aplicando el PCGE”. Lima: Grupo Acrópolis.
Consejo de Normas Internacionales de Contabilidad - IASB (2009). "NIIF para las PYMES”. Londres: El Consejo.

Effio Pereda, Fernando (2011). "NIIF para las PYMES”. Lima: Entrelíneas S.R.L.

García Quispe, José Luis (2012). "NIIF para PYMES”. Lima: Instituto Pacífico S.A.C.

Valdivia Loayza, Carlos. (2010). "Diferencias de las NIIF para PYMES con las NIIF completas". Ponencia en XXII Congreso Nacional de Contadores Públicos del Perú. Arequipa.

Vilchez, Percy; Pignatta, Alfredo (2011). "Aplicación práctica de la NIIF para las PYMES”. Ponencia en la XXIX Conferencia Interamericana de Contabilidad 2011. Puerto Rico: Asociación Interamericana de Contabilidad. 